Geophysical and Astrophysical Fluid Dynamics

Vol. 00, No. 00, 00 Month 2012, 1-17

\title{
Convection driven by temperature and composition flux with the same diffusivity
}

\author{
J. A. Whitehead* \\ MS\#21, Physical Oceanography Department, \\ Woods Hole Oceanographic Institution, Woods Hole, MA 02543, USA \\ (Received 00 Month 20xx; final version received 00 Month 20xx)
}

\begin{abstract}
Temperature, pressure, and composition determine density of fluids within the earth, the ocean, our atmosphere, stars and planets. In some cases, variation of composition component $C$ competes equally with temperature $T$ to determine buoyancy-driven flow. Properties of two-dimensional cellular convection are calculated with density difference between top and bottom boundaries determined by difference of temperature $T$ (Dirichlet boundary conditions, quantified by Rayleigh number $R a$ that is positive destabilising), fluxes of $C$ (Neumann boundary conditions quantified by Raf that is positive stabilising), and Prandtl number $P r$. Numerical solutions in a 2-dimensional rectangular chamber are analysed for Prandtl numbers $\operatorname{Pr}=1, \infty$. For $R a$ and $R a f>0$ and $R a f$ above approximately 300, subcritical instability separates $T$-driven convection from $C$-dominated stagnation. The flow is steady but a sudden change in $R a$ or $R a f$ produces decaying pulsations to the new flow. A boundary layer solution for rapid flow exists in which $T$, which has the Dirichlet condition, is more sensitive to flow speed than $C$ with the Neumann condition. A new type of pulsating flow occurs for $R a$ and $R a f<0$. The pulsations are characterised by slow flow with gradually strengthening compositional plumes in a thermally stratified flow interrupted by rapid flow with gradually weakening compositional plumes. In this slow speed range, $C$ is more sensitive to speed than $T$.
\end{abstract}

Keywords: Double component convection; mixed boundary conditions; subcritical instability; pulsations

\section{Introduction}

All the large fluid bodies on earth, on some planets, on their moons, and in stars have more than one component contributing to density that drives or constrains the motion. Atmospheric air has temperature and moisture, ocean water has temperature and salinity, magmas and parts of the mantle have temperature and composition, the Earth's core has temperature and composition, planets and their moons have temperature and assorted components, the sun has temperature and the helium/hydrogen ratio, and some stars have the ratio of two or more elements with hydrogen.

The multi-component nature of the resulting flows is important and has led to development of specialised studies (e.g. double diffusion in the ocean, magmas, and stars, moist convection in the atmosphere, the dynamics of crystalline laden suspensions in magmas and liquid metals, and the behaviour of hot turbidity flows in volcanic plumes). Double diffusion, in which two components affecting density are distinguished from each other only by their two values of diffusivity, is probably the most intensively studied example. In those problems, Soret and Dufour effects (the migration of one component driven by changes in the other component) are ignored, and only the two different diffusivities, and hence the two diffusion time scales dynamically define each substance. However, in many cases within nature, the two components are not different because of internal processes, but because they are driven by different boundary conditions. For example the ocean surface temperature closely follows atmospheric

\footnotetext{
*Email: jwhitehead@whoi.edu
} 
temperature but the changes in salinity are driven by a flux of water across the ocean surface through evaporation, precipitation and land run-off. The temperature/salinity composition is a fundamental property of the ocean and it is linked to abrupt transitions of the thermohaline circulation regime (Broecker et al. 1985, Boyle 1990, Keigwin and Jones 1994, Keigwin, et al. 1994, Bard et al. 1996, Broecker 1997, Stocker and Wright 1991, Burns et al. 2003, Weart 2003, and many others). The abrupt transitions are understood to arise as a direct consequence of different $T$ and $S$ time scales (Stommel 1961, Bryan 1986, Cessi 1994, Rahmstorf 1995, Manabe and Stouffer 1995, Rahmstorfand Ganopolski 1999, Whitehead 1998, Weaver et al. 1999, Hearn and Sidhu 1999, Bulgakov and Skiba 2003). In atmospheric circulation models, land and ocean surface temperatures are specified along with evaporative water flux. In cooling magmas or in metallurgy, the boundary $T$ remains fixed at the solidification temperature but composition flux is determined by solidification processes. Early theoretical studies and laboratory studies of flows with different $T$ and $C$ boundary conditions arose in studies of the melting of ice in salt water, alloy formation and magma modeling(Huppert and Turner 1981) but these generally focused on double diffusion effects (e.g. Turner 1973, Welander 1989, Tsitverblit and Kit 1993, Tsitverblit 1995).

In all of the "structured" models used to study these issues, (e.g. models with a specified internal structure such as box models or numerical models of ocean circulation with many internal processes parameterised), the internal mixing properties of $T$ and $C$ are set equal to each other so that double-diffusion or phase changes within the fluid are not relevant. An emphasis on the effect of the different boundary conditions alone in pure fluid dynamics problems was initiated by setting the diffusion of $T$ and $C$ equal (Tsitverblit 1997a,b, 2004, 2007, Zhao et al. 2007). Tsitverblit (2007) describes these problems as "a fundamentally new class of hydro-dynamic instabilities underlying the formation of spatial and temporal flow patterns from a steady equilibrium state of spatially homogeneous fluid."

Some laboratory experiments illustrate the changes back and forth between $T$ and $C$ dominated flows driven by different boundary conditions. A chamber exposed to a temperature difference and a flux of salt water pumped steadily into a fresh water environment recovers the abrupt transitions and hysteresis of the original Stommel box model and a slightly more complex chamber produces a severely limited hysteresis range (Whitehead 2009). In other experiments, spontaneous oscillations back and forth between temperature and salinity driven flows occur (Whitehead et al. 2005).

This study presents some new results for the classical case of cellular convection driven by two components with two different types of boundary conditions and the same diffusivity. In this study, we call the second component "composition" rather than salinity or some other dissolved substance. Either the fluid has a warmer temperature below with a stabilising upward flux of composition, or a colder temperature below with a destabilising downward flux of composition. This is the configuration studied by Tsitverblit (2004), but over much wider parameter ranges. The objective is to find overall the basic properties since so many natural systems possess two types of boundary conditions. A square two-dimensional chamber contains fluid. A temperature difference is imposed between the top and bottom boundaries quantified by Rayleigh number $R a$. A laterally uniform flux of composition $C$ into or out of the chamber is imposed along the top and bottom boundaries quantified by the flux Rayleigh number $R a f$. Therefore temperature $T$ is driven by Dirichlet boundary conditions and composition $C$ is driven by Neumann boundary conditions. Zero flux boundary conditions are imposed on the lateral boundaries with free-slip conditions on all four boundaries. Therefore, this represents one convection cell in a periodic array in the horizontal direction. Both infinite Prandtl number and $\operatorname{Pr}=1$ cases are investigated with numerical calculations over a wide range of positive and negative values of $R a$ and $R a f$.

URL: http:/mc.manuscriptcentral.com/ggaf Email: andrew.soward@newcastle.ac.uk 


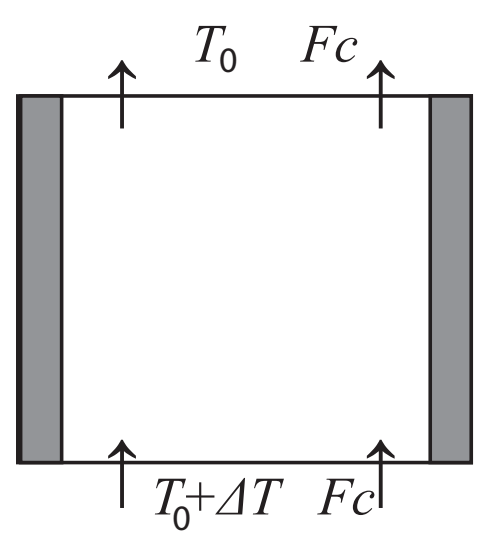

Figure 1. A rectangular container with constant temperature along the top, elevated temperature along the bottom and a uniform upward flux of composition. There is zero flux of heat and composition through the sides.

\section{Procedure}

The Boussinseq equations of motion, which govern the flow, are

$$
\begin{aligned}
\nabla^{\prime} \cdot \boldsymbol{u}^{\prime} & =0 \\
\rho_{0} \frac{\partial \boldsymbol{u}^{\prime}}{\partial t^{\prime}}+\rho_{0} \boldsymbol{u}^{\prime} \cdot \nabla^{\prime} \boldsymbol{u}^{\prime} & =\nabla^{\prime} p^{\prime}+\mu \nabla^{\prime 2} \boldsymbol{u}^{\prime}+g \alpha \rho_{0} T^{\prime} \widehat{\boldsymbol{k}}-g \beta \rho_{0} S^{\prime} \widehat{\boldsymbol{k}} \\
\frac{\partial T^{\prime}}{\partial t^{\prime}}+\boldsymbol{u}^{\prime} \cdot \nabla^{\prime} T^{\prime} & =\kappa \nabla^{\prime 2} T^{\prime} \\
\frac{\partial C^{\prime}}{\partial t^{\prime}}+\boldsymbol{u}^{\prime} \cdot \nabla^{\prime} C^{\prime} & =\kappa \nabla^{\prime 2} C^{\prime} .
\end{aligned}
$$

In them, $\boldsymbol{u}^{\prime}$ is the velocity vector (prime denotes it is dimensional), $T^{\prime}$ is temperature, $\widehat{\boldsymbol{k}}$ is the unit vector in the direction of gravity directed downward in the $z^{\prime}$ coordinate direction and $t^{\prime}$ is time. The constants are average density $\rho_{0}$, the viscosity $\mu$, the acceleration due to gravity $g$, the linear coefficient of thermal expansion $\alpha$, the linear coefficient of composition expansion $\beta$, and the thermal and composition diffusivity both $\kappa$. The fluid is in a square chamber of depth $D$ and width $W=D$ (figure 1). The average initial temperature of the fluid is $T_{0}$ and the temperature along the prescribed boundary is raised to a temperature distribution with size $T_{0}+\delta T$ at $t^{\prime}=0$. Simultaneously, the average initial composition is zero and the composition flux $F c^{\prime}=-\kappa\left[\partial C^{\prime} / \partial z^{\prime}\right]$ is imposed along the prescribed boundary.

To non-dimensionalise, the velocity scale is set to $\kappa / D$, the scale of temperature in deviation from $T_{0}$ is $\Delta T$, the composition scale is determined by the absolute magnitude of the derivative of composition imposed at the boundary $\Delta C=D\left[\partial C^{\prime} / \partial z^{\prime}\right]$, and time scale is $D / \kappa$. Henceforth, all dimensionless symbols are unprimed. Temperature and composition obey

$$
\begin{aligned}
& \frac{\partial T}{\partial t}+\boldsymbol{u} \cdot \nabla T=\kappa \nabla^{2} T, \\
& \frac{\partial C}{\partial t}+\boldsymbol{u} \cdot \nabla C=\kappa \nabla^{2} C .
\end{aligned}
$$

The equation governing the vorticity $\zeta=\partial u / \partial z-\partial w / \partial x$ for two-dimensional right-handed Cartesian flow is

$$
\left[\frac{1}{P r}\left(\frac{\partial}{\partial t}+\boldsymbol{u} \cdot \nabla\right)-\nabla^{2}\right] \zeta=R a \frac{\partial T}{\partial x}-R a f \frac{\partial C}{\partial x}
$$


and the equation for the stream function $\psi$, where $u=\partial \psi / \partial z$ and $w=-\partial \psi / \partial x$ is

$$
\nabla^{2} \psi=\zeta
$$

The three governing dimensionless numbers are the Rayleigh number $R a=g \alpha(\Delta T) D^{3} / \kappa \nu$, the composition flux Rayleigh number $R a f=-g \beta\left[\partial C^{\prime} / \partial z^{\prime}\right] / \kappa \nu$ and the Prandtl number $\operatorname{Pr}$.

Although a numerical bifurcation and stability analysis with spatial spectral discretisation might be used to study this problem, we decided to use a finite-difference approach. It is applicable to all parameter ranges without the need for new formulas at each bifurcation point of the study. The flow, temperature, and composition distributions are numerically calculated in a rectangular grid of points. Free slip boundary conditions are imposed on all four sides. Lateral flux of heat and composition are set to zero through the two side walls. The top and bottom boundary conditions are $T=0$ across the top boundary and $T=1$ along the bottom. The composition flux is set to 1 along both the top and bottom. Therefore, this represents a single cell representing a periodic solution to the flow. Standing waves might exist, but laterally propagating periodic flow patterns are excluded. Additional computations with periodic side conditions would be required to include them but are not included in this study. For $R a$ and $R a f>0$, the chamber (figure 1) has bottom temperature $\Delta T$ above the top temperature, and composition flux $F_{C}$ is upward and positive. The analysis advances the values on the grid forward in time. This highly tested method produces results over any desired wide range of parameters at the expense of greater precision that could be obtained using more specialised approaches in limited parameter ranges. Initially, there is no motion and linear $T$ and $C$ profiles are specified in the interior to match the boundary conditions. Equations $(2 \mathrm{a}, \mathrm{b})$ are advanced numerically using a leapfrog-trapezoidal scheme for each time step $\delta t$. Then, (3) is solved either by stepping ahead in time as in (2) or, in the case of infinite $\mathrm{Pr}$, using a standard Poisson equation solver with zero vorticity satisfied at all the boundaries. Then, (4) is solved using the Poisson solver with zero streamfunction at the boundaries.

Calculations at the four boundaries for flux using conventional second one-sided difference approximations in conjunction with the flux boundary conditions conserve $C$ to the order of the numerical accuracy. The rectangular grid is two-dimensional with 128 points on a side giving an accuracy of 1 part in $10^{6}$. Resolution was tested as in an earlier study (Whitehead et al. 2013) and all extrema of the fields are accurate to better than 1\%. Since $C$ has only flux boundary conditions, there is a slow random drift in the spatially averaged value of concentration $C$ within the chamber over long times. If one-sided difference equations are used to calculate the flux into the system, the drift is of the order of the finite difference errors that are a few parts in $10^{-6}$. This drift is decreased by many orders of magnitude by using a better method. An external line of grid points is added outside each line of boundary grid points. This corresponds to a thin layer of solid around the chamber and in this layer the desired value of flux into the chamber is imposed by pure diffusion. Therefore, the variations of the value of flux into and out of the chamber occur at the numerical truncation order of $10^{-17}$. Consequently, $C$ randomly fluctuates about the mean to $\mathrm{O}\left(10^{-16}\right)$ (figure 2). Heat flow is also much more accurately preserved with this method since the sides are thermally insulated to the order of $10^{-17}$.

\section{Results}

\subsection{Heated from below, stabilizing flux of composition}

If we artificially set $C=0$ everywhere in the grid, there are well known flows. For example, the classical Rayleigh (1916) stability problem applies to this, and a boundary layer solution exists for a two-dimensional cell (Turcotte and Schubert 2002). With two components, the study by Tsitverblit (2004) shows that subcritical instability exists for Raf sufficiently large 


\section{Geophysical \& Astrophysical Fluid Dynamics}

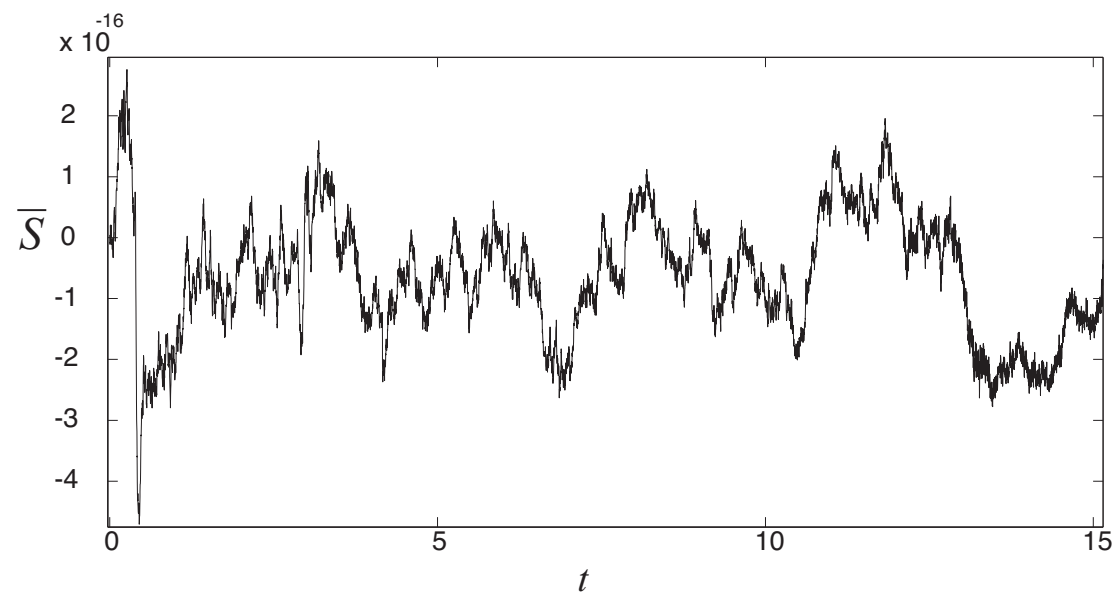

Figure 2. The value of mean composition remains at about one order of magnitude above the digital truncation level over a great span of time. This was obtained from the run shown in figure 3.

compared to $R a$.

To determine stability of a flow, a calculation is started with a linear initial temperature and composition distribution. Temperature has the value 1 on the bottom that decreases linearly up to zero at the top, and composition has the same linear change but starts with the value 0.5 on the bottom and ends with -0.5 on the top. The initial state has exactly zero flow except that variations in each value of $T$ and $C$ at grid points exist at the truncation noise level of $\mathrm{O}\left(10^{-17}\right)$. This noise establishes a perturbation to the static fields. If $R a$ is large enough to make a disturbance linearly unstable, the perturbation grows. Whether the perturbation grows or decays is easily viewed by subtracting out the original linear distributions of $T$ and $C$. In all cases, flows have the same qualitative structure irrespective of grid size and only the quantitative values change a little. This result is also found for plume studies (Whitehead et al. 2013). Benchmark calculations with different grid sizes (in parenthesis) and a time step of $10^{-6}$ with $R a f=0$ give the four critical values $R a c=805$ (32), 791 (64), 782 (128), and 780 (256). The critical value from linear theory for constant temperature free slip Rayleigh-Benard convection in a square box, is $R a c=2^{3} \pi^{4}=779.2727$. Since the value of $R a c$ using the 128 grid is within $1 \%$, this study reports results using the 128 grid. Therefore, the extrema for all fields are accurate to better than $1 \%$.

With a small value of Raf added, the growth of a small disturbance continues to be exponential in time. However, at larger Raf, the growth is oscillatory and exponential as shown in figure 3 for the maximum of the absolute value of streamfunction $|\psi|_{\max }$.

Three types of runs determine critical parameter values and amplitudes. First, for very tiny amplitudes (e.g. before $t=8$ in figure 3) many successive runs are used to locate the largest possible value of $R a$ that has the fields decay in time. This is the critical Rayleigh number $R a c$ for fixed $R a f$ and $P r$. The value of $R a c$ is readily located by spanning successive values of $R a$. An example for evolution of growing and decaying oscillations at two slightly different values of $R a$ for small $\left(10^{-7}\right)$ amplitude is shown in figure 4 . Values of $R a c$ are rounded off to the nearest ten to be consistent with numerical accuracy and are shown in Table 1.

The second type of run starts with steady flow at sufficiently large $R a$ and then sequentially decreases $R a$ by small amounts (e.g. figure 5 ) and waits for a sufficient time for amplitude to reach a steady value. The maximum absolute value of streamfunction for four values of Raf and two Prandtl numbers are shown in figure 6 . Subcritical instability is very small but detected for $R a=300$, and it clearly occurs for $R a f=10^{3}$ and $10^{4}$. The minimum Rayleigh number for flow with a subcritical instability is called Ram, and values are shown in Table 1. 


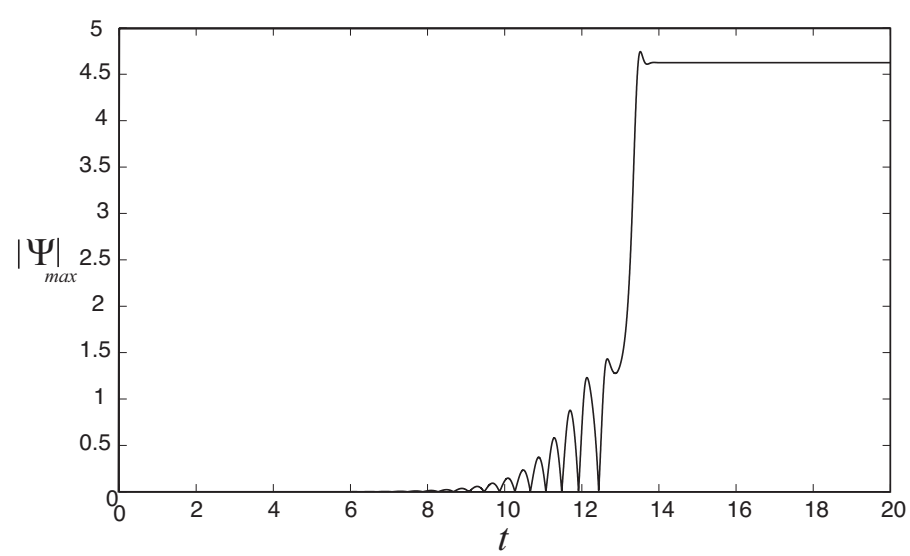

Figure 3. Evolution of the streamfunction for $R a=2200, \operatorname{Raf}=1000, \operatorname{Pr}=\infty$.

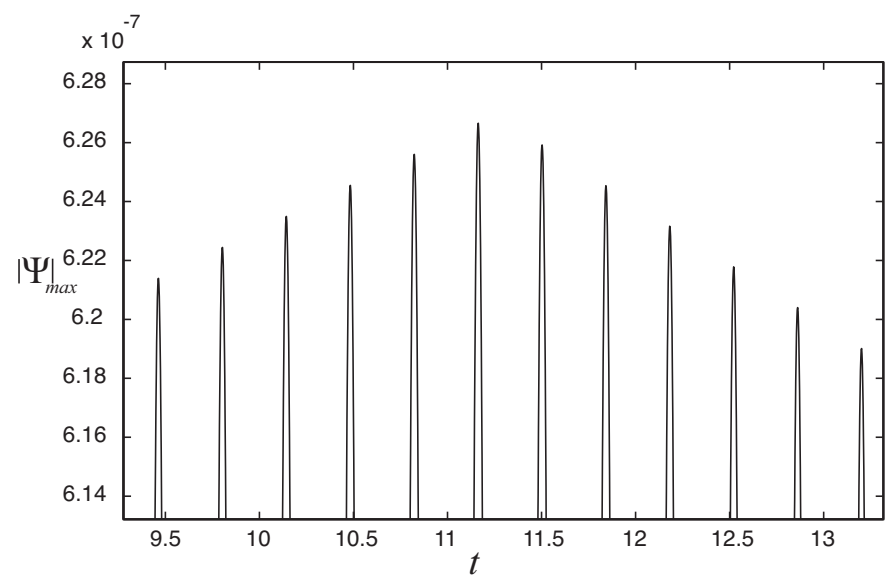

Figure 4. The peak amplitude of streamfunction versus time with two values of $R a$ spanning $R a c$. In the interval $9.3<t<11.3, R a=2098$ and the peaks increase with time. In the interval $11.3<t<13.3, R a=2097$ and the peaks decrease with time. $\operatorname{Raf}=1000, \operatorname{Pr}=\infty$.

\begin{tabular}{|r|c|c|c|c|}
\hline$R a f$ & $R a c(P r=1)$ & $R a c(\operatorname{Pr}=\infty)$ & $\operatorname{Ram}(\operatorname{Pr}=1)$ & $\operatorname{Ram}(\operatorname{Pr}=\infty)$ \\
\hline 90 & 940 exp & 940 exp & $X$ & $X$ \\
300 & 1270 exp & 1270 exp & 1260 & 1260 \\
1000 & 2470 osc & 2100 osc & 1660 & 1660 \\
$10^{4}$ & 9510 osc & 10600 osc & 6310 & 6620 \\
\hline
\end{tabular}

Table 1. Values of the critical and minimum Rayleigh numbers and whether the growth of the very small flows is exponential or oscillatory.

For $R a=90$ subcritical instability is not found.

The third type of run determines the unstable points (dashed lines in figure 6) using interrupted calculations. First, a calculation is started with $R a$ large enough to develop steady convection. Then, $R a$ is reduced to a value below $R a m$ and $\left|\psi_{\max }\right|$ decays. When it reaches a desired value, $R a$ is changed so that it and $\left|\psi_{\max }\right|$ are close to a dashed line. After a short adjustment, if the magnitude is above the dashed line, $\left|\psi_{\max }\right|$ grows, and if it is below the dashed line, it decreases. 


\section{Geophysical \& Astrophysical Fluid Dynamics}

Geophysical and Astrophysical Fluid Dynamics

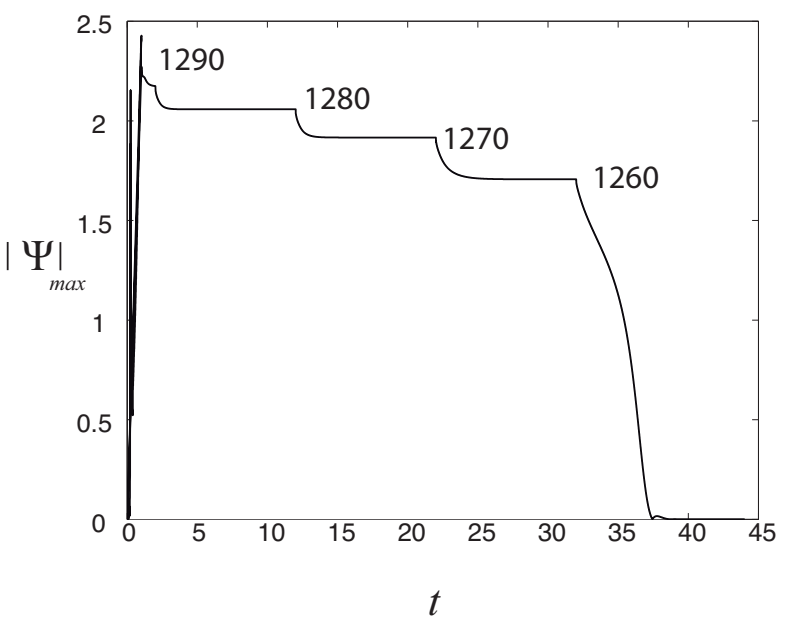

Figure 5. An example of the determination of successive values of the maximum value of streamfunction as $R a$ is slightly stepped down to the shown values. $\operatorname{Raf}=300, \operatorname{Pr}=\infty$

(a)
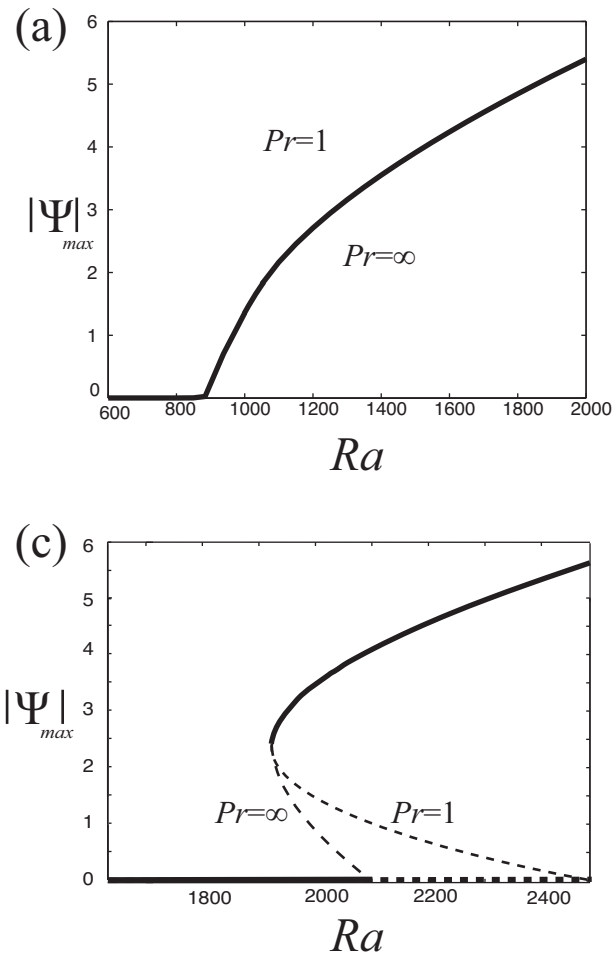

(b)

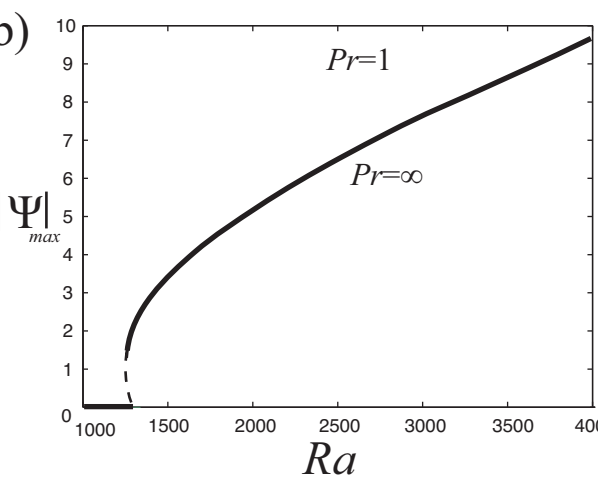

(d)

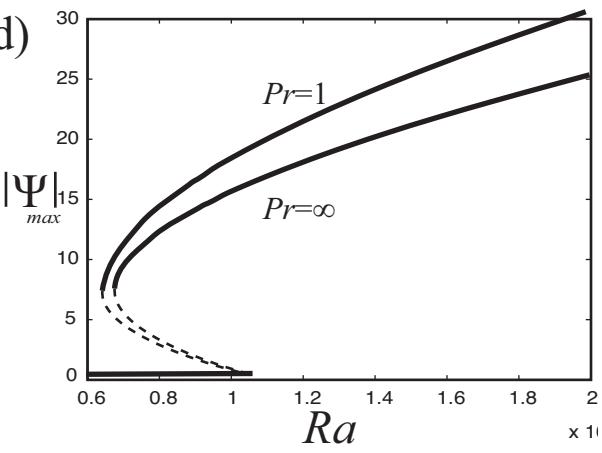

Figure 6. Speed versus the Dirichlet variable $R a$ for the Neumann variables: (a) $R a f=90$, (b) $R a f=300$, (c) $R a f=1000$, and (d) $R a f=10,000$ and for both $\operatorname{Pr}=1$ and $\operatorname{Pr}=\infty$.

\subsection{Large amplitude flow}

For $R a=R a f=10^{4}$ and for both cases with $\operatorname{Pr}=1$ and $\operatorname{Pr}=\infty$, the temperature and composition distributions develop boundary layer structures. A typical example is shown in figure 7. The boundary layers bend at the corners to form $T$ and $C$ plumes along the sides that convey them vertically. The relative magnitudes in the plumes illustrate the effect of the two different boundary conditions. Temperature difference between the isothermal interior 

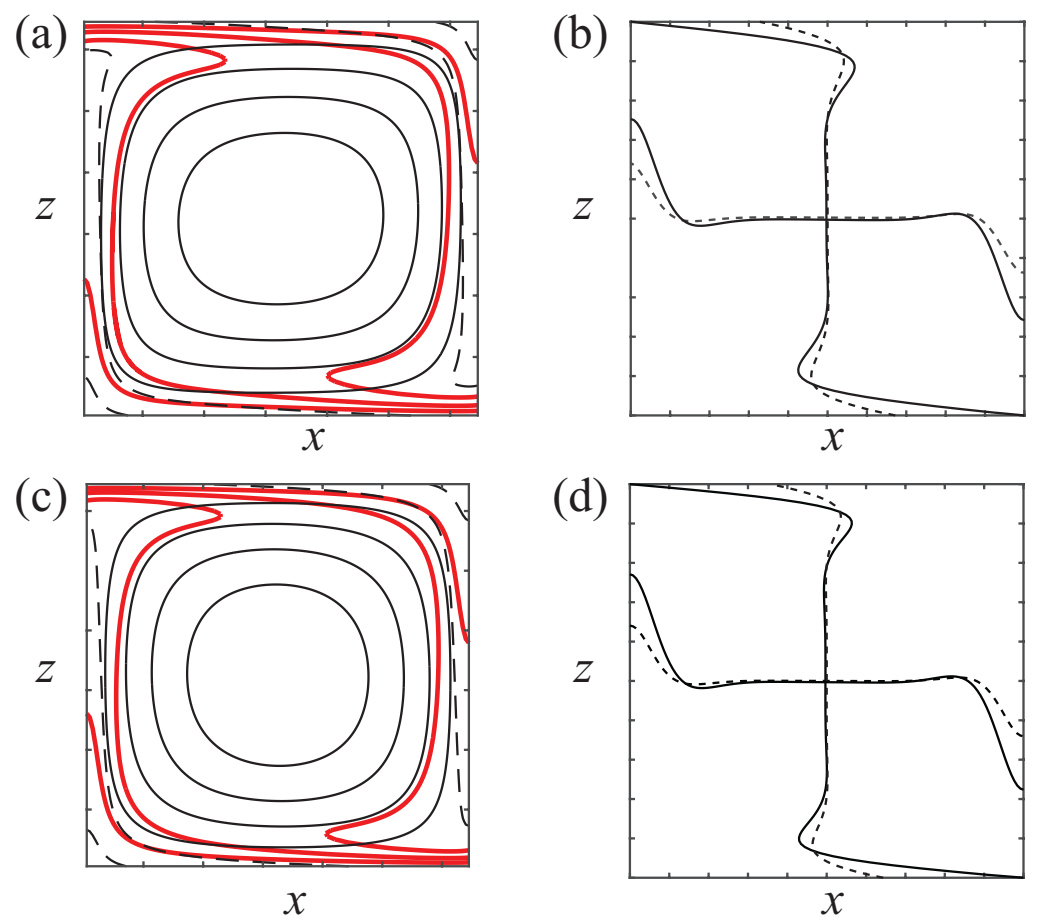

Figure 7. Temperature and composition for steady flow in a chamber that is one dimensionless unit in depth and width with $R a=R a f=10^{5}$ and for $\operatorname{Pr}=\infty(\mathrm{a}, \mathrm{b})$ and for $\operatorname{Pr}=1(\mathrm{c}, \mathrm{d})$. (a,c) Vertical section showing isotherms (every $T=0.2$, heavy red curves), composition (every $C=0.02$, dashed lines) and steamfunction (solid contours every 0.2 units between $\left|\psi_{\max }\right|$ and zero). (b,d) Mid-cell vertical and horizontal profiles of $T$ (solid) and of $5(C+0.5)$ (dashed). (Colour online)

and each plume has magnitude 0.25 and the component difference is approximately ten times smaller. The flow pattern is almost the identical for both $\operatorname{Pr}=1$ and $\operatorname{Pr}=\infty$, except that the flow with large $\mathrm{Pr}$ is approximately $10 \%$ slower and accompanied by a slightly greater value $C$ and smaller value of $T$. These results suggested that it is feasible to develop the boundary layer solution in section 4 .

\subsection{Stabilising temperature and destabilising composition flux}

With $R a$ and $R a f$ set to negative values, composition flux drives the convection and heat flux stabilises it. (For negative values, we multiply the symbol by -1 . Therefore, its value is positive and we avoid confusion with inequality signs). With $R a=0$, for example, the critical value of $-R a f$ is 470.6234 . The stability and flow characteristics for a small value of $R a$ such as $-R a=10^{2}$ are not remarkable since a perturbation simply grows asymptotically to a steady flow. Subcritical instability is absent. However, new behaviour exists for both $R a$ and $R a f>10^{4}$ (figure 8(a)). A range of pulsations separates flow with 1 cell from flow with 2 cells. The amplitude of the flow pulsates (faster and slower). There is no hysteresis, amplitude of a pulsation is the same irrespective of how the values of $R a$ and $R a c$ are changed to get to the final parameter. For example, with $-R a f=4 \times 10^{5}$, the range with pulsations separates a steady boundary layer driven flow at $R a<3 \times 10^{4}$ from a layered stratified flow with two vertical cells at $R a>2 \times 10^{5}$. Pulsations within such a range are also present at $-R a f=10^{5}$, $8 \times 10^{5}$ and $10^{6}$. Amplitude of the flow at $-R a f=4 \times 10^{5}$ reflects the three distinct regions (figure $8(\mathrm{~b})$ ).

The three regions have different flow structures. The 1 cell flow has a boundary layer structure similar to the flow in figure 7 for all the cases shown in this figure. Naturally, the density 


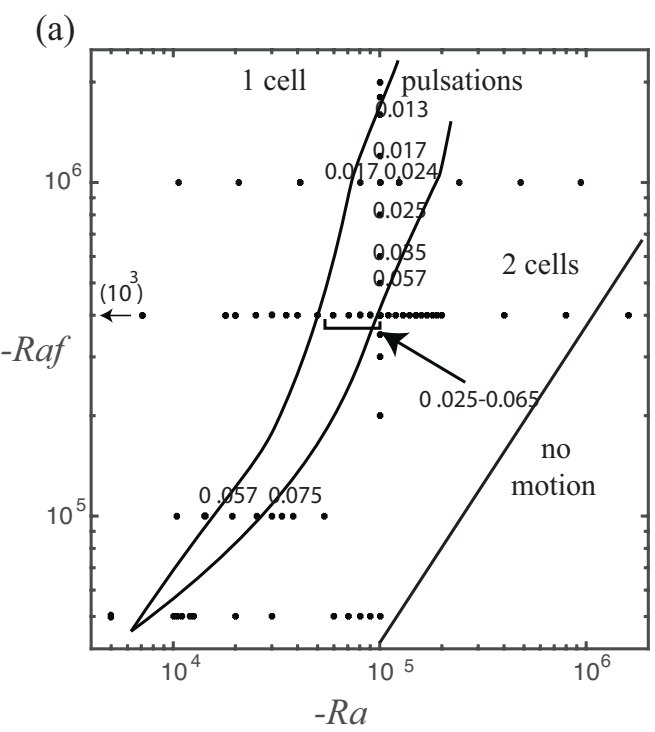

(b)

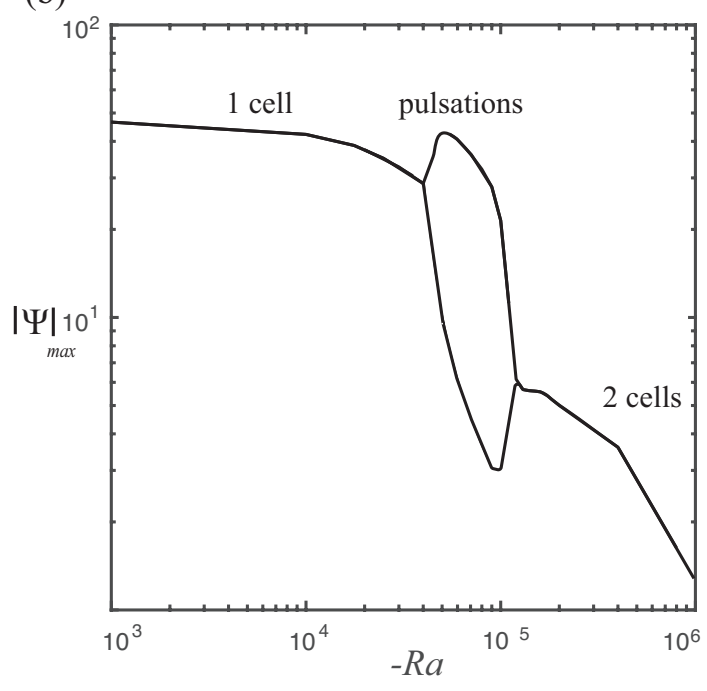

Figure 8. Summary of results for $\operatorname{Pr}=\infty$. (a) Phase diagram of investigated values with the regions of different modes indicated. Numbers show the periods of pulsations. (b) The maximum absolute value of streamfunction for calculations with - Raf $=4 \times 10^{5}$. If a value of Ra has two values of maximum absolute value of streamfunction, a pulsation exists and the greatest and smallest of the maximum over time is shown.

difference within the plumes is obviously dominated by $C$ rather than $T$. For pulsations, the boundary layer flow speeds up and slows down at the smaller values of $R a$ in the pulsation range. For example, along the line with $-R a=10^{5}$ all the pulsations are like this. However, at larger $R a$ (e.g. near the 2 cell transition), the flow oscillates back and forth between flow with 1 cell and flow with 2 cells. The flow patterns during one cycle with alternating cells are shown in figure 9. Panels show: (a) At the beginning of a cycle, there are two cells at the two corners where the $C$ plumes extend vertically from the horizontal surfaces. (b) These cells flow slowly enough to cause the composition in the boundary layer to gradually increase. (c) Soon thereafter, the plume density increases and this speeds everything up. (d) Faster flow produces one cell that is similar to the boundary layer flow shown previously in figure 7 . (e) This rapid flow however causes a weakening of the concentration in the boundary layer and this weakening is greater than weakening of $T$. (f) This soon decreases density in the plumes and causes the single cell to divide into two slower cells. Then, the cycle repeats. One can summarise the shapes of the central contours of streamfunction in this sequence as peanut-oval-circle-square-and peanut again.

The "two cells" region of figure 8 has one convection cell along the top and another along the bottom (figure 10). This begins to resemble a layered flow. Within the range of parameters where two cells exist, at greater values of $-R a$ the cells become more localised to the top and bottom. At even greater values, flow ceases as the two cells vanish. For example at $R a f>10^{6}$, all flow has vanished at $-R a=1.5 \times 10^{6}$, all flow has vanished at $-R a=1.5 \times 10^{6}$.

\section{Analysis}

\subsection{Pulsations}

The simplicity of the flow patterns allow one to construct simple idealised models of the flow. Figure 11 shows mid-level grid point values for the differences (extreme left minus extreme right) of $T\left(=T^{\prime}\right)$ and $C^{\prime}$ during a pulsation. First, the difference of $C^{\prime}$ follows the difference 

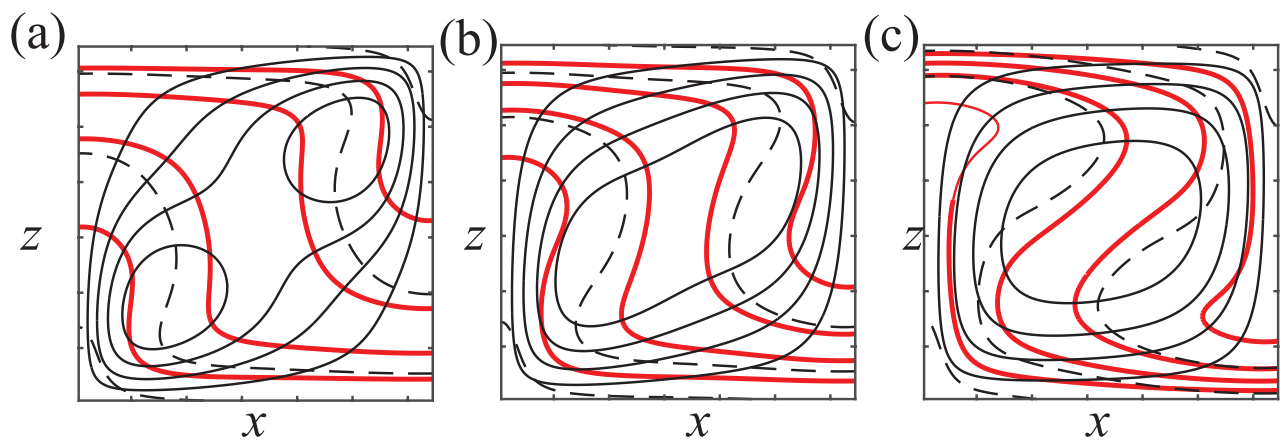

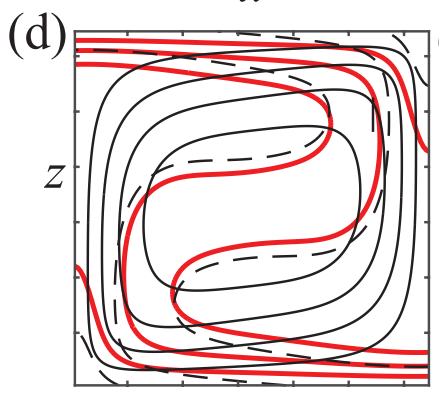

$x$ (e)

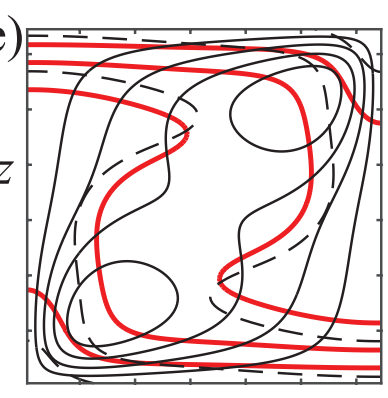

$x$ (f)

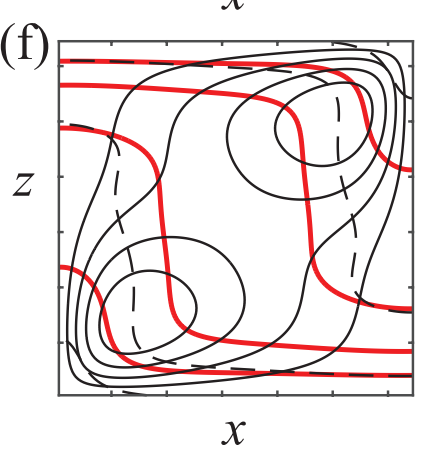

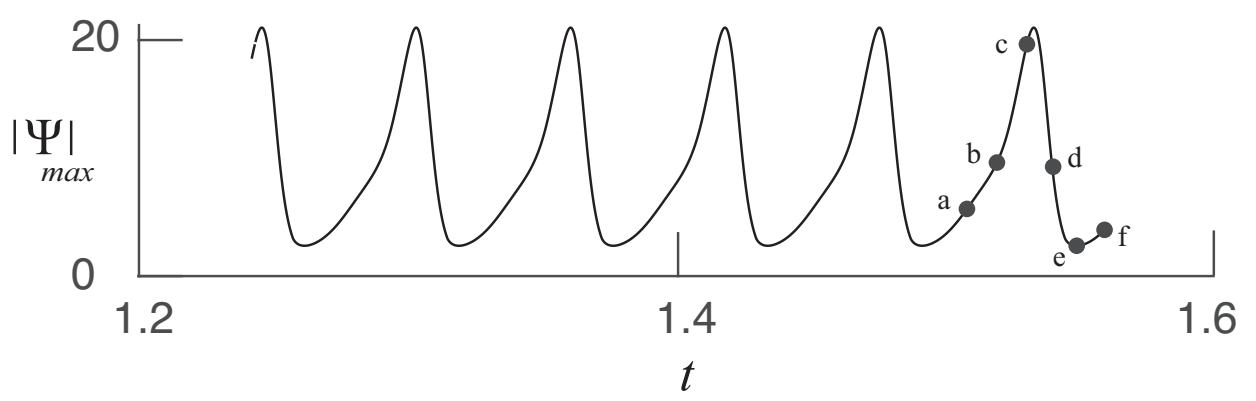

Figure 9. (Top and middle rows) Contours of maximum absolute value of streamfunction (solid), $T$ (heavy red) and $C$ (dashed) during the peanut-oval-circle-square-peanut pulsation sequence. Times are indicated in the bottom graph. $-R a=10^{5},-R a f=4 \times 10^{5}, \operatorname{Pr}=\infty$. (Colour online)

of $T^{\prime}$. Second, the difference of $C^{\prime}$, which is driving the flow, is almost perfectly in phase with the streamfunction value.

A simple way to picture why $C^{\prime}$ follows $T^{\prime}$ is to start with a model of a parcel of fluid that rotates around the edges of the convection cell and thus encounters alternating values of $T$ and a flux of $C$ at the outer edge. Consider the well-known thermal conduction solution of $T$ from a sinusoidal time-varying temperature at $y=0$ next to a half-space. We take the variation to be in a $y$ direction away from this boundary to avoid confusion with other sections. The solution is (Turcotte and Schubert 2002, eq. 4-89)

$$
T=T_{0}+\Delta T \exp \left(-y \sqrt{\frac{\omega}{2 \kappa}}\right) \cos \left(\omega t-y \sqrt{\frac{\omega}{2 \kappa}}\right) .
$$

In our case, the gradient of $C$ obeys the same differential equation. Therefore, the integral in $y$ gives $C$ as

$$
\left.C=C_{0}+\frac{\partial C}{\partial y}\right]_{y=\infty} \sqrt{\frac{\kappa}{\omega}} \exp \left(-y \sqrt{\frac{\omega}{2 \kappa}}\right) \cos \left(\omega t-y \sqrt{\frac{\omega}{2 \kappa}}-\frac{\pi}{4}\right) .
$$

This shows how $T$ follows $C$, as expected and it demonstrates that generally the two are 
Figure 10. Contours of maximum absolute value of streamfunction (solid), $T$ (heavy red) and $C$ (dashed) for layered steady flow at $-R a=-R a f=4 \times 10^{5}$. (Colour online)

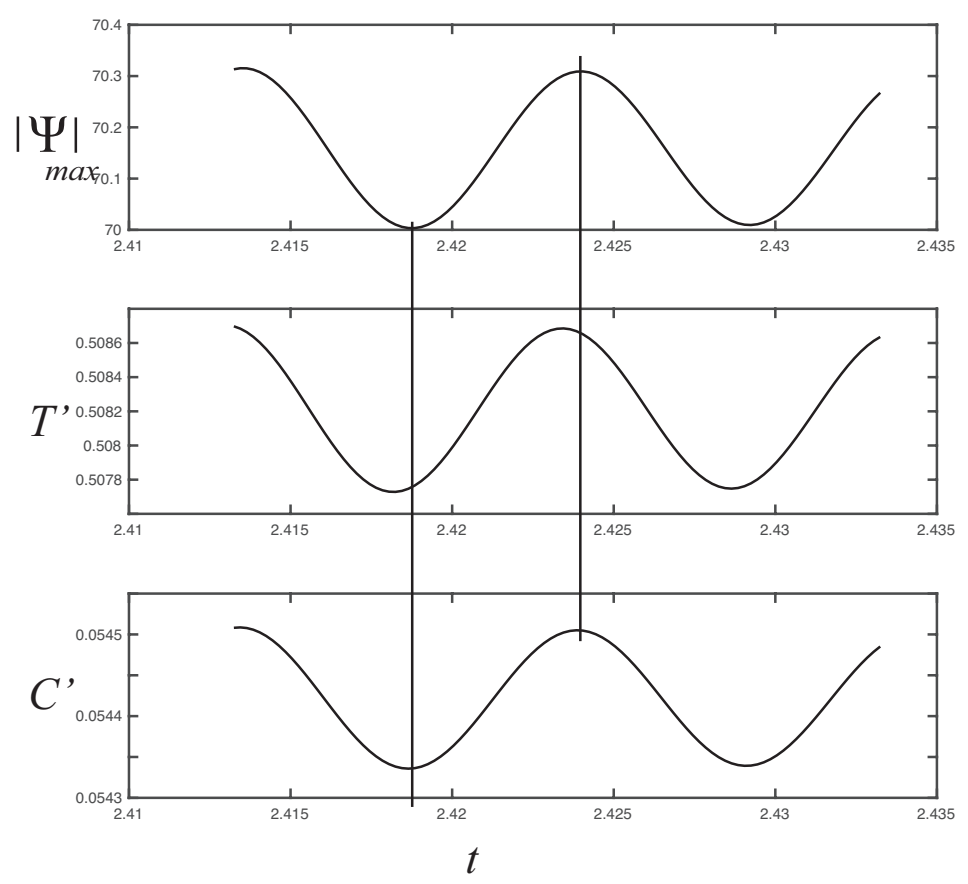

Figure 11. Maximum absolute value of streamfunction (top), $T$ difference (middle) and $C$ difference (bottom) during a pulsation cycle. $-R a=10^{5},-$ Raf $=1.2 \times 10^{6}$.

not in phase for any pulsations at any flow rate. Naturally, this phase difference leads to the oscillation properties of this flow.

\subsection{Boundary layer theory}

Let us construct a model of a convection cell driven by the boundary layers shown in figure 7 . For simplicity, we modify the model of a convection cell from Section 6.21 of Turcotte and Schubert (2002) starting from our equations (2)-(4). The single cell has dimensionless length 
$L=\lambda^{\prime} / 2 D^{\prime}$ (the pair of cells has dimensional length $\lambda^{\prime}$ ). A uniform velocity $u_{0}$ in the positive $x$-direction exists across the top of this cell and a uniform velocity in the opposite direction exists across the bottom. Along the sides, the vertical velocity $w_{0}$ is uniform and upward on the left $(x=0)$ and downward (negative) and uniform on the right $(x=L)$. The temperature at the bottom $(z=0)$ is $T=1$ and at the top $(z=1)$ is $T=0$. The flux of $C$ is 1 in the upward direction and this flux is uniform across both the bottom and top surfaces.

First the temperature field is calculated. The interior of the cell has closed circulation and the isothermal core has the average temperature $T=0.5$. A developing cold thermal boundary layer moves toward the right along the top and a developing warm thermal boundary layer moves toward the left along the bottom. Therefore, a plume of sinking cold fluid forms along the right side of the cell and a plume of rising warm fluid forms along the left side of the cell. The boundary layer temperature profile across the top is found by assuming uniform translation of fluid across the top with the fluid initially at $T=0.5$. Immediately under the top boundary, $T$ obeys the well-known error function (Turcotte and Schubert 2002, eq. 6-347)

$$
T_{\text {top }}=0.5 \operatorname{erfc}\left(\frac{1-z}{2} \sqrt{\frac{u_{0}}{x}}\right) .
$$

The temperature distribution along the bottom is similar but it results from flow in the opposite direction and has higher temperature. It is

$$
T_{\text {bottom }}=1-0.5 \operatorname{erf}\left(\frac{z}{2} \sqrt{\frac{u_{0}}{L-x}}\right),
$$

where erf and erfc are respectively the error and complimentary error functions. The top boundary layer turns clockwise $90^{\circ}$ at the upper right corner and forms a sinking cold plume. Likewise, the bottom boundary layer turns clockwise $90^{\circ}$ at the lower left corner and forms a rising hot plume. We now estimate the total power $P_{T}$ (rate of energy production) generated by buoyant thermal forces. It is given in dimensionless form by Ra times vertical velocity times density variation from the temperature deviation from its average value

$$
P_{T}=R a \int_{0}^{1} \int_{0}^{L} w(T-0.5) \mathrm{d} x \mathrm{~d} z .
$$

The principle contribution to $P_{T}$ arises within the ascending and descending plumes. The plumes are also the principle contributors to vertical heat flux away from the boundaries, and since there is no internal heat generation in the fluid, the vertical heat flux is

$$
H f_{T}=\int_{0}^{L} w T \mathrm{~d} x
$$

and it is independent of $z$ except near the bottom and top boundary layer regions. Since in this model it is assumed that vertical velocity is constant along the sides of the cell, any temperature profile can be used to determine the entire vertical integral of a plume and thereby evaluate $(2 \mathrm{a}, \mathrm{b})$ throughout the interior. It is simplest to use the temperature profiles of the temperature fields that exist just before they turn the corner. As the fluid turns the bottom corner, for example, the vertical coordinate $z$ in the boundary layer transforms to the lateral coordinate $x^{\prime \prime}=u_{0} z / w_{0}$. Thus the body force in the ascending plume is (as in Turcotte and Schubert 2002, eq. 6-354)

$$
\begin{aligned}
f_{b T} & =R a \int_{0}^{\infty} \operatorname{erfc}\left(\frac{w_{0} x^{\prime \prime}}{2 u_{0}}\left(\frac{u_{0}}{L}\right)^{1 / 2}\right) \mathrm{d} x^{\prime \prime} \\
& =2 R a \frac{u_{0}}{w_{0}}\left(\frac{L}{u_{0}}\right)^{1 / 2} \int_{0}^{\infty} \operatorname{erfc} z \mathrm{~d} z=2 R a \frac{u_{0}}{w_{0}}\left(\frac{L}{\pi u_{0}}\right)^{1 / 2} .
\end{aligned}
$$


The power driven by buoyancy is found by multiplying $f_{b T}$ times the vertical velocity $w_{0}$ over the depth $(=1)$. A similar value is calculated from the top boundary layer that turns the corner and forms a descending plume. Therefore, total power from (9) is

$$
P_{T}=4 R a \sqrt{u_{0} L / \pi} .
$$

The contribution to the total power from concentration flux $P_{C}$ is easy to calculate in a similar manner. The magnitude of the power is

$$
P_{C}=\operatorname{Raf} \int_{0}^{1} \int_{0}^{L} w(x, z)(C(x, z)-\bar{C}(z)) \mathrm{d} x \mathrm{~d} z .
$$

The upward $C$ flux per unit cell length equals 1, therefore a cell of length $L$ has total upward $C$ flux equal to $L$. Concentration $C$ forms structures similar to the thermal field, including boundary layers, plumes and an interior with a uniform value of $C$. For positive $R a f$, the vertical work hinders convection and the power consumed is $P_{C}=-L R a f$. The total power is the sum of power from the two fluxes

$$
P_{\text {total }}=4 R a \sqrt{u_{0} L / \pi}-L R a f .
$$

This is balanced by frictional dissipation. In this simplified model of viscous flow, the velocity field has pure shear, so that

$$
u=u_{0}(2 z-1), \quad w=w_{0}(1-2 x / L) .
$$

The stress on the bottom is $2 u_{0}$ per unit length and the dissipation at both bottom and top is equal to 2 times lateral velocity times stress times the length or $4 u_{0}^{2} L$. The stress on the side is $2 w_{0} / L$ and the stress for two sides times velocity is $4 w_{0}^{2} / L$. Using the equation of continuity $\left(u_{0} / L\right)+w_{0}=0$, this becomes $4 u_{0}^{2} / L^{3}$.

The power generation is equal to frictional dissipation and this gives the relation

$$
4 R a \sqrt{\frac{u_{0} L}{\pi}}-\operatorname{Raf} L=4 u_{0}^{2}\left(L+\frac{1}{L^{3}}\right) .
$$

For $R a f=0$, this reduces to eq. 6-362 of Turcotte and Schubert (2002)

$$
u_{0}=\frac{L^{7 / 3}}{\left(1+L^{4}\right)^{2 / 3}}\left(\frac{R a}{4 \sqrt{\pi}}\right)^{2 / 3}
$$

and for large values of $R a$ and $R a f$

$$
u_{0 \min }=\frac{\pi L R a f^{2}}{16 R a^{2}} .
$$

Solutions to (16) are visualised by plotting the right and left sides as a function of $u_{0}$ (figure 12). The right side is a parabola extending upward from zero and the left side is a parabola on its side. For $\operatorname{Raf}>0$, the left side increases from a negative value. Solutions are intersection of such curves and they have a number of features:

1. For a fixed value of $R a$, there is one value of $R a f$ that corresponds to the minimum on the intersection curves. For Raf with a value greater than that, there is no solution.

2. Lower values of $R$ af have two intersections.

3. The intersection to the right is stable. A perturbation of smaller velocity there would follows a light curve lying above the dark curve. This produces more power release than dissipation and the consequent acceleration will increase velocity and cause a return to the intersection. A perturbation of a larger velocity also stabilises by leading to less power release than dissipation. The deceleration produces slower flow and a return to the intersection. 

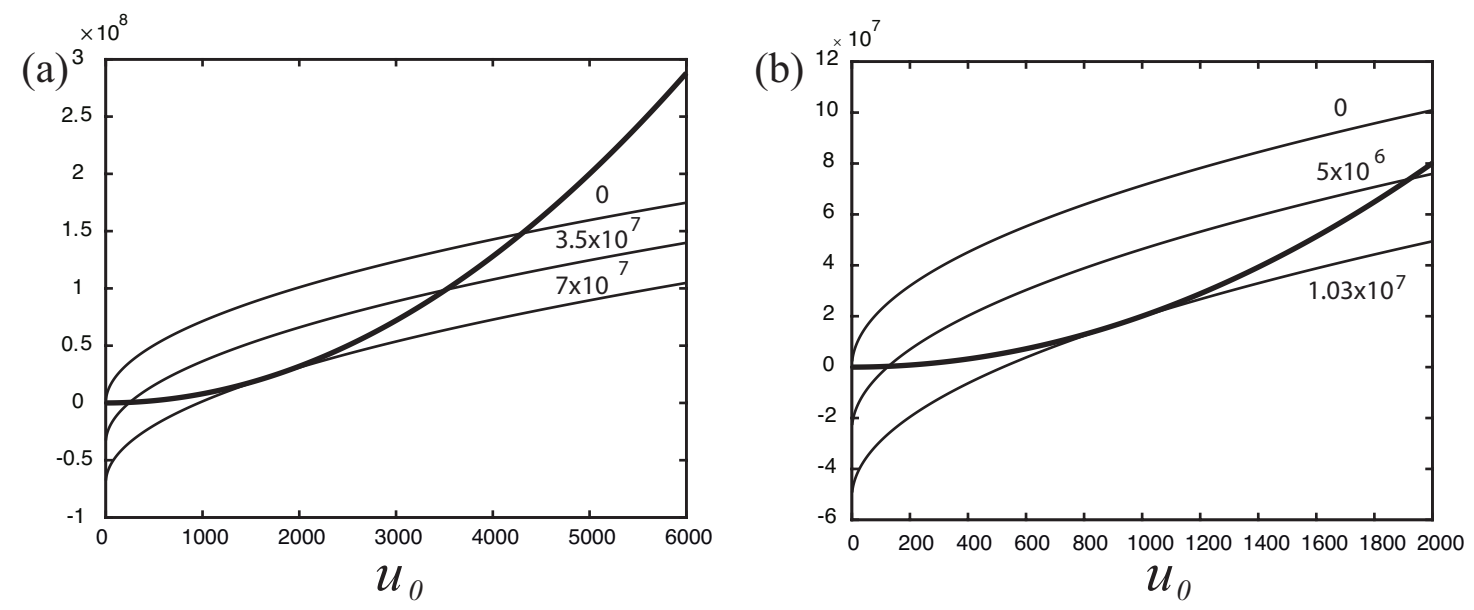

Figure 12. (a) The values of the right and left sides of (16) for $R a=10^{6}$ and $L=1$. The solutions are intersections. The single thick parabola curving upward comes from the right hand side of (16). The three other curves come from the left-hand side with three values of $R a f$ as shown. (b) The same for $L=5$.

4. The intersection to the left is unstable. The arguments are exactly opposite to case 3 .

5. Convection driven by composition $(R a f<0)$ has light curves originating above zero. One solution exists and (16) indicates that wide cells are preferred.

The intersection of the two curves where there is only one value denotes the critical minimum value $R a m$ for any $R a f$. If $R a$ is less than this value, no intersection is possible. The minimum value is found by rearranging (16) as

$$
4 R a \sqrt{\frac{u_{0} L}{\pi}}-4 u_{0}^{2}\left(L+\frac{1}{L^{3}}\right)=\operatorname{Raf} L
$$

and noting that the term on the left has a maximum value with respect to $u_{0}$. Setting the derivative to zero, the velocity is

$$
u_{0}=\frac{L^{7 / 3} R a^{2 / 3}}{(16 \pi)^{1 / 3}\left(1+L^{4}\right)^{2 / 3}}
$$

and this gives a minimum value of $R a$

$$
\operatorname{Ram}=\sqrt{\frac{2 \pi}{L}}\left(1+L^{4}\right)^{1 / 4}\left(\frac{R a f}{3}\right)^{3 / 4} .
$$

To compare these equations against the numerical results, first, comparison is made between (16) and results from numerical runs over the range $R a=30,000$ to $10^{6}$ with $R a f=10^{5}$. Analysis of many flows like the one shown in figure $7(\mathrm{a}, \mathrm{b})$ found that the metric $5|S|_{\max }$ represents the peak velocity in the entire cell very closely. Therefore, $u_{0}=5|S|_{\max }$ is plotted versus $R a$ in figure 13. Then, these values are used in (16) to calculate a value of $R a$ to generate a curve for the boundary layer theory results. The comparison is not quantitatively close; the actual and calculated values of Ra lie approximately a factor of 5 from each other. This rather poor comparison probably reflects that the model is very simple. Possibly the approximation of uniform boundary velocity in the theory makes much more robust boundary layers than convection with stress free boundaries does. Better quantitative comparison is found on a log-log plot since the numerical results do approach the slope $2 / 3$ corresponding to the limit with $R a f=0$ (17), which is a well-known result (Turcotte and Schubert 2002). Note that the results in figure 13 show that convection cell properties show little effect from compositional drag except near the transition region. 


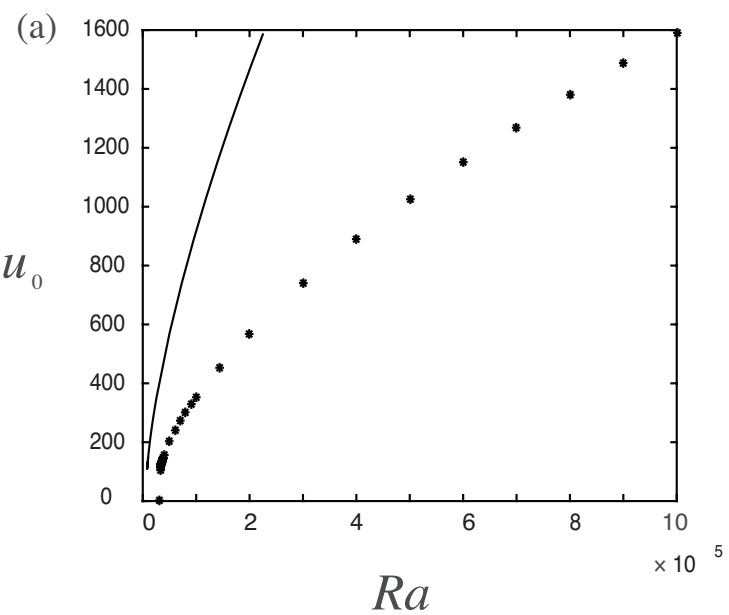

(b)

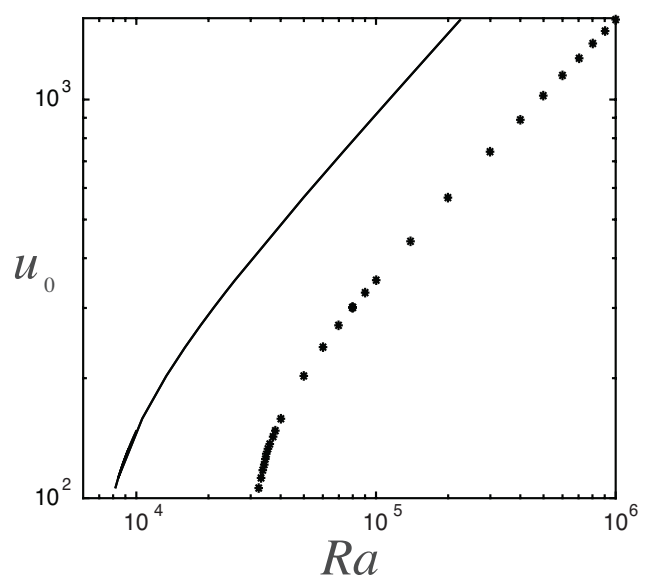

Figure 13. Velocity from numerical runs (dots) and from equation (18) (solid line) with $R a f=10^{5}$ and $L=1$. (a) Linear, (b) Log-log. The lightly dashed line is for Raf $=0$ (17) and it has been extended down to (21).

A second test for the termination of the solution at small $R a$ using (21) also does not quantitatively compare well with numerical runs. The numerical run sequence in figure 13 had a termination of motion at $R a=32,000$ while (21) gives $\operatorname{Ram}=7353$.

\section{Summary and discussion}

Finite amplitude instability and nonlinear oscillations occur for a convection cell driven by the two different boundary conditions. For positive $R a$ and $R a f$, the overall qualitative results are the same as found by Tsitverblit (2004). There is a range of subcritical instabilities if $R a f$ is large enough. However, the quantitative agreement is not perfect. For example, Tsiverblit found the onset of subcritical instability for $\operatorname{Pr}=6.7$ at $R a \sim R a$ to lie in the range $R a=$ 120 to 240 , but the present results produce an almost undetectable subcritical instability at $R a=300$ for the two cases $\operatorname{Pr}=1, \infty$. Also, we find that with $\operatorname{Raf}=10000$, and $\operatorname{Pr}=1$, $R a c$ is 9600 whereas in Tsiverblit states that $R a c$ goes to infinity for $\mu=R a f R a \rightarrow 1$. These differences between present and earlier quantitative values might be due to a number of factors:

1) Different values are given for $\mathrm{Pr}$.

2) Cell wavelength differs. 
J. A. Whitehead

3) A very accurate method of numerically incorporating the flux condition is used here.

Finally, the subcritical instability resembles the subcritical instability in double diffusion (Veronis 1964), but the exact overlap remains to be analysed. The boundary layer solution gives a useful perspective on the role of the two different boundary conditions. With positive $R a f$, the speed abruptly ceases with decreasing $R a$. The cut-off value does not have close quantitative agreement with Ram from numerical runs.

The parameter range $R a$ and $R a f<0$ produces pulsations for $-R a>4 \times 10^{4}$. The pulsations differ from the oscillations at marginal stability in double diffusion (Veronis 1964, Turner 1973) and with multiple boundary conditions, (Welander 1989, Tsitverblit 2007), which occur for $R a>0$ with stabilising salinity present and in some thermohaline laboratory experiments (te Raa 2001, Whitehead et al. 2005). They are also unlike the binary fluid oscillations (Matura and Lücke 2006) that are travelling waves. These occur in experiments in a layer with zero lateral velocity imposed at the top and bottom and apparently are linked to the Soret effect. We conducted a small number of runs with cells eight times greater than the depth with both positive and negative large values of $R a$ and $R a f$ and did not find travelling waves, but the range of parameters studied so far is very small. To investigate a link with the travelling oscillations in binary fluids, a study incorporating cells with periodic lateral boundary conditions with and without the Soret and Dufor effects would be a useful new project.

The pulsations that are driven by two different boundary conditions seem to be absent in double diffusion studies and are therefore new. One can imagine that the stabilising temperature field is a spring that aids the pulsation. Faster flow compresses the spring but slowly produces less compositional driving. Slower flow relaxes the spring and slowly produces more compositional driving. The resulting phase differences produce the oscillation. The pulsation period is a small fraction of the scaling timescale, so such pulsations might potentially arise in evaporative basins driven by salinity differences such as the Mediterranean Sea or the Red Sea. Like salt fingers, the new pulsations are dynamical structures with their own particular set of balances that may fruitfully be studied further.

The present study has admittedly sacrificed numerical precision so that wide ranges of the governing parameters can be explored. The more accurate approach for a boundary layer flow solution by Vynnycky and Masuda (2013) might produce better quantitative agreement with the numerical results, but it requires more computation and was not followed. Although the present boundary layer solution does not give close quantitative comparison with numerical results, it has a simplicity that allows it to be used for teaching and communicating the dynamics to those in other disciplines.

\section{References}

Bard, E., Hamelin, B., Arnold, M. Montaggioni, L., Cabiochparall, G., Faure, G. and Rougerie, F., Deglacial sea-level record from Tahiti corals and the timing of global meltwater discharge. Nature 1996, 382, $241-244$. Boyle, E.A., Quaternary deepwater paleoceanography. Science 1990, 249, 863-870.

Broecker W.S., Thermohaline circulation, the Achilles heel of our climate system: Will man-made $\mathrm{CO}_{2}$ upset the current balance?. Science 1997, 278, 1582-1588.

Broecker, W.S., Peteet, D.M. and Rind, D., Does the ocean-atmosphere system have more than one stable mode of operation?. Nature 1985, 315, 21-26.

Bryan, F., High-Latitude composition effects and interhemispheric thermohaline circulations. Nature 1986, 323, 301-304.

Bulgakov S.N. and Skiba, Y.N., Are transitions abrupt in Stommel's thermohaline box model?. Atmósfera 2003, 16, 205-229.

Burns, S.J., Fleitmann, D., Matter, A., Kramers, J. and Al-Subbary, A.A., Indian ocean climate and an absolute chronology over Dansgaard/Oeschger events 9 and 13. Science 2003, 201, 1365-1367.

Cessi, P., A simple box model of stochastically forced thermohaline flow. J. Phys. Oceanogr. 1994, 24, 19111920.

Hearn, S.J. and Sidhu H.S., The Stommel model of shallow coastal basins. Proc. Roy. Soc. London A 1999, 


\section{Geophysical \& Astrophysical Fluid Dynamics}

455, 3997-4011.

Huppert, H.E. and Turner, J.S., Double-diffusive convection. J. Fluid Mech. 1981, 106, 299-329.

Keigwin, L.D., Curry, W.B., Lehman, S.J. and Johnsen, S., The role of the deep-ocean in North-Atlantic climate-change between 70-Kyr and 130-Kyr ago. Nature 1994, 371, 323-326.

Keigwin, L. . and Jones, G.A., Western North-Atlantic evidence for millennial-scale changes in ocean circulation and climate. J. Geophys. Res. 1994, 99(C6), 12,397-12,410.

Manabe, S. and Stouffer, R.J., Simulation of abrupt climate change induced by freshwater input to the North Atlantic Ocean. Nature 1995, 378, 165-167.

Rahmstorf, S., Bifurcations of the Atlantic thermohaline circulation in response to changes in the hydrological cycle. Nature 1995, 378, 145-149.

Rahmstorf, S. and Ganopolski, A., A simple theoretical model may explain apparent climate instability. J. Climate 1999, 12, 1349-1352.

Rayleigh, L., LIX. On convection currents in a horizontal layer of fluid, when the higher temperature is on the under side. The London, Edinburgh, and Dublin Philosophical Magazine and Journal of Science 1916, 32(192), 529-546.

Stocker T.F. and Wright, D.G., Rapid transitions of the ocean's deep circulation induced by changes in surface water fluxes. Nature 1991, 351, 729-732.

Stommel H., Thermohaline convection with two stable regimes of flow. Tellus 1961, 3, 224-230.

Matura, P. and Lücke, M., Driving convection in a fluid layer by a temperature gradient or a heat current. Phys. Rev. E 200673 (3), 037301.

te Raa, L., Convective oscillations in a laboratory model. GFD Fellows Report 2001, http://gfd.whoi.edu/proceedings/2001/PDF/rep_teraa.pdf.

Tsitverblit, N. and Kit, E., The multiplicity of steady flows in confined double-diffusive convection with lateral heating. Phys. Fluids A: Fluid Dynamics 1993, 5 (4), 1062-1064.

Tsitverblit, N., Bifurcation phenomena in confined thermosolutal convection with lateral heating: Commencement of the double-diffusive region. Phys. Fluids 1995, 7 (4), 718-736.

Tsitverblit, N.A., The finger regime of double-diffusive convection with the equal diffusion coefficients and mixed boundary conditions. Double-Diffusive Processes 1996 Summer Study Program in Geophysical Fluid Dynamics, 1997a, Woods Hole Oceanographic Institution, 145.

Tsitverblit, N.A., On the nature of direct instabilities in double-component convection with different boundary conditions. Phys. Fluids 1997b, 9(8), 2458-2460.

Tsitverblit, N., Multiplicity of equilibrium states in laterally heated thermosolutal systems with equal diffusivity coefficients. Phys. Fluids 1999, 11 (9), 2516-2538.

Tsitverblit, N., Mechanism of finite-amplitude double-component convection due to different boundary conditions. Phys. Lett. A 2004, 329 (6), 445-450.

Tsitverblit, N., Double-component convection due to different boundary conditions in an infinite slot diversely oriented to the gravity. Ann. Phys. 2007, 322 (8), 1727-1770.

Turcotte, D.L., and Schubert, G., Geodynamics. Cambridge University Press, New York 2002, 456 pp.

Turner, J. S., Buoyancy effects in fluids. Cambridge University Press, New York, 1973, 367 pp.

Veronis, G., On finite amplitude instability in thermohaline convection. J. Mar. Res. 1964, 23, 1-17.

Vynnycky, M. and Masuda, Y., Rayleigh-Bénard convection at high Rayleigh number and infinite Prandtl number: Asymptotics and numerics. Phys. Fluids 2013, 25 (11), 113602.

Weart, S., The discovery of rapid climate change. Phys. Today 2003, 56 (8), 30-36.

Weaver, A.J., Bitz, C.M., Fanning, A.F. and Holland, M.M., Thermohaline circulation: High-latitude phenomena and the difference between the Pacific and Atlantic. Annu. Rev. Earth Planet Sci. 1999, 27, 231-285.

Welander, P., A new type of double diffusive instability. Tellus 1989, 41A, 66-72.

Whitehead, J. A., Multiple T-S states for estuaries, shelves and marginal seas. Estuaries 1998, 21, 278-290.

Whitehead, J. A., Abrupt transitions and hysteresis in thermohaline laboratory models. J. Phys. Oceanogr. 2009, 39, 1231-1243.

Whitehead, J.A., Cotel, A., Hart, S., Lithgow-Bertelloni, C. and Newsome, W., Numerical calculations of two-dimensional large Prandtl number convection in a box. J. Fluid Mech. 2013, 729, 584-602.

Whitehead, J.A., te Raa, L., Tozuka T., Keller, J.B. and Bradley K., Laboratory observations and simple models of slow oscillations in cooled salt-stratified bodies. Tellus 2005, 57A (5), 778-809, doi:10.1111/j.16000870.2005.00150.x.

Zhao, F.Y., Liu, D. and Tang, G.F. Multiple steady flows in confined gaseous double diffusion with discrete thermosolutal sources. Phys. Fluids 2007, 19 (10), 107103. 